\title{
Analysis of the Results of Gastrin-17 and its Related Influencing Factors in Health Check-Up Population
}

\section{Yan Shen}

Healthcare Department, Union Hospital, Tongji Medi

Junchao Zeng

Healthcare Department, Union Hospital, Tongji Medi

\section{Sanping Xu}

Healthcare Department, Union Hospital, Tongji Medi

Rui Yang ( $\nabla$ yangrui5245@163.com )

Healthcare Department, Union Hospital, Tongji Medical College, Huazhong Universi

\section{Research article}

Keywords: Gastrin 17, Pepsinogen, Helicobacter pylori, Inflammatory factors

Posted Date: September 21st, 2021

DOl: https://doi.org/10.21203/rs.3.rs-921083/v1

License: (9) This work is licensed under a Creative Commons Attribution 4.0 International License. Read Full License 


\section{Abstract}

Background: To analyze the difference of serum gastrin- 17 levels in different sexs, ages, and body mass index (BMI) of healthy people, and to explore the correlation between gastrin 17 and pepsinogen, in addition to study the influence of Helicobacter pylori infection and various inflammatory factors on the secretion level of gastrin-17.

Methods: 531 subjects who received physical examination in our center from April 2019 to December 2019 were enrolled in the study. All the staff were tested for gastrin 17 (G17), pepsinogen I (PGI), pepsinogen II (PGII), PGI / PGII (PGR), Helicobacter pylori (Hp) and C-reactive protein (CRP) and other inflammatory factors. To compare the difference of $\mathrm{G} 17$ secretion in different populations and its correlation with PG, then to understand the HP infection and the influence of inflammatory indicators on G17.

Results: There was no significant difference in the secretion level of $\mathrm{G} 17$ in different sex, age and BMI ( $P$ $>0.05)$; G17 was positively correlated with PGI and PGII, but negatively correlated with PGR; the level of G17 in Helicobacter pylori positive patients was $10.16 \pm 12.84$, which was significantly higher than that in negative patients(3.27 \pm 6.65$), P=0.017,95 \% \mathrm{Cl}: 1.713(1.100,2.668)$; the increase of serum amyloid $\mathrm{A}(\mathrm{SAA})$ in different inflammatory indicators was the high-risk factor of $\mathrm{G} 17$ abnormality, $P=0.016,95 \% \mathrm{Cl}$ : $2.692(1.202,6.028)$, obviously CRP and erythrocyte sedimentation rate (ESR) had no effect on G17 abnormalities.

Conclusions: The secretion of G17 is closely related to PG and HP. Combined screening is helpful for early screening of gastrointestinal diseases in healthy or high-risk groups of gastric cancer, but the influence of inflammatory indicators on G17 should be excluded to improve the reliability of the results.

\section{Background}

Gastric cancer is a common digestive tract malignant tumor. Its incidence rate and mortality rate rank the forefront of global malignant tumors ${ }^{[1]}$. It is the final result of the progression of precancerous lesions, including non-atrophic gastritis, atrophic gastritis, intestinal metaplasia and dysplasia. Epidemiological studies show that the 5-year survival rate of early gastric cancer is as high as $90 \%$, while that of advanced gastric cancer was only $25 \%{ }^{[2]}$. Therefore, early screening, early diagnosis and early treatment are important measures to reduce the mortality of gastric cancer.

Compared with the markers in tissues, serological markers are less invasive, easier to obtain, lower cost and shorter time-consuming. Currently available serological markers associated with gastric diseases include Pepsinogen I(PGI), Pepsinogen II(PGII), PG I/PG II(PGR), Gastrin 17(G17) and anti-Helicobacter pyliri (HplgG). Based on the existing evidence, these serological markers may help to identify those with precancerous lesions who should be referred to gastroscopy ${ }^{[3]}$. Although the use of these biomarkers of the stomach for serological biopsy of gastric mucosa has been used for the detection of gastric cancer 
and precancerous lesions for more than 20 years, previous studies mainly focused on PGI, PGI / II ratio and HplgG, while other biomarkers such as G-17 and PGIl were ignored ${ }^{[4]}$.

G17 is closely related to inflammation, immunity, invasion and metastasis of gastric cancer cells. It has a low level of expression in healthy people. When the pathological changes of gastritis, gastric cancer and others occur, the level of the expression increases significantly ${ }^{[5]}$. The increase or decrease of $\mathrm{G} 17$ can prompt the occurrence risk of gastric cancer. The increase or decrease of G17 can prompt the occurrence risk of gastric cancer. It is not a direct cause of gastric cancer in itself, but it can promote some carcinogenic factors, activate multiple signal transduction pathways, produce anti apoptotic and antiinflammatory effects, and induce gastric acid secretion, thus leading to tumorigenesis ${ }^{[3,6]}$. The aim of this study was to analyze the G17 findings and possible influencing factors of normal physical health people, in order to understand the secretory characteristics and application value of G17 in healthy people.

\section{Methods}

1. Participants: a total of 531 healthy subjects who received physical examination in the healthcare department center of Wuhan Union Hospital from April 2019 to December 2019 were selected for the study, including 307 males and 224 females, aged 22-78 years. In addition to routine physical examination, G17, PGI, PGII, PGR, Hp typing, C-reactive protein(CRP), erythrocyte sedimentation rate(ESR) and serum amyloid $\mathrm{A}(\mathrm{SAA})$ were detected. Excluding acute and chronic inflammatory disease, liver and gallbladder disease, diabetes, blood disease, kidney disease, rheumatic immune disease, tumor, etc. Grouped according to age, BMI and Hp typing.

\section{Methods}

2.1 Serological test: About $3 \mathrm{ml}$ fasting venous blood was collected, after centrifugation, serum was obtained. The serum PGI, PGII, PGR and G17 were quantitatively detected by enzymelinked immunosorbent assay (ELISA). The testing equipment is BIOHIT Healthcare(Hefei) Co., Ltd and Wuhan Yolins Technology Trade Co., Ltd. According to the requirements of the kit, PGI < 70ng / $\mathrm{ml}$ concurrently with $\mathrm{PGR}<3$ were defined as $\mathrm{PG}$ positive, and $\mathrm{G} 17<1$ or $>15 \mathrm{pmol} /$ I was regarded as $\mathrm{G} 17$ positive.

$2.2{ }^{13} \mathrm{C}$ breath test: The test was carried out on an empty stomach in the early morning. The equipment used in the research is the kit produced by Shenzhen Zhonghe Headway Bio-Sci囚Tech Co., Ltd, along with the HCBT- $02{ }^{13} \mathrm{C}$ breath test instrument. The positive standard of $\mathrm{Hp}$ infection was 4.0 times higher than the reference value.

2.3 Inflammatory index detection: CRP was measured by immunofluorescence quantitative detection. The apparatus used was I-CHROMA Rester immunofluorescence analyzer provided by Boditech Med Inc; ESR was calculated by Westergren method; SAA adopts SIEMENS BN prospect specific protein analyzer as well as matching reagents, strictly according to the operation manual. 
2.4 Statistical methods: Spss19.0 statistical software was used for analysis. T test was used in the comparison between the two groups, while the One-way ANOVA was used for multiple groups. Correlation analysis was performed by Spearman rank correlation test, with calibration level of 0.01 . The single factor Logistic regression analysis was used to analyze the influencing factors. $P<0.05$ was statistically significant.

\section{Results}

1. The difference of gastrin 17 secretion in different sex, age, and BMI

There was no significant difference in the secretion level of $\mathrm{G} 17$ between male and female, the same as different age groups and BMI groups $(P>0.05)$ (Table 1$)$.

Table 1

The difference of gastrin 17 secretion in different sex, age and $\mathrm{BMI}$

\begin{tabular}{|lllll|}
\hline Groups & $\mathbf{N}$ & $\mathbf{X} \pm \mathbf{s}$ & t/F & $\boldsymbol{P}$ \\
\hline Sex & & & & \\
\hline male & 307 & $5.32 \pm 10.11$ & 1.204 & 0.299 \\
\hline female & 224 & $6.38 \pm 9.85$ & & \\
\hline Age & & & & \\
\hline$\leq 30$ & 39 & $5.01 \pm 10.68$ & 2.178 & 0.055 \\
\hline $31-40$ & 86 & $4.17 \pm 8.13$ & & \\
\hline $41-50$ & 198 & $4.81 \pm 7.40$ & & \\
\hline $51-60$ & 164 & $7.66 \pm 12.73$ & & \\
\hline $61-70$ & 39 & $6.58 \pm 11.11$ & & \\
\hline$\geq 70$ & 5 & $8.65 \pm 6.15$ & & \\
\hline BMl & & & & \\
\hline$\triangle 24$ & 174 & $5.35 \pm 8.74$ & 0.606 & 0.546 \\
\hline $24-27.9$ & 146 & $6.60 \pm 12.35$ & & \\
\hline$\geq 28$ & 58 & $5.70 \pm 7.84$ & & \\
\hline
\end{tabular}

2. Correlation analysis between G17 and PG 
G17 and PG were significantly correlated, $P=0.000$, G17 was positively correlated with PGI and PGII ( $\mathrm{r}=$ 0.322 and 0.452$)$, but negatively correlated with PGR $(r=-0.367)$ (Table 2).

Table 2

Correlation analysis between $\mathrm{G} 17$ and PG

\begin{tabular}{|llll|}
\hline Serum markers & Median & $\mathbf{r}$ & $\boldsymbol{P}$ \\
\hline PGI & $57.49(3.3-17.65)$ & 0.322 & 0.000 \\
PGII & $9.80(2.5-41.3)$ & 0.452 & 0.000 \\
PGR & $6.63(0.37-16.83)$ & -0.367 & 0.000 \\
\hline
\end{tabular}

3. Secretion of G17 in Hp infected population

The secretion of G17 in HP infected population $(10.16 \pm 12.84)$ was significantly higher than that in uninfected (3.27 \pm 6.65$)$ (Table 3$)$.

Table 3

Secretion of G17 in Hp infected population

\begin{tabular}{|c|c|c|c|c|}
\hline Hp & $\mathbf{N}$ & $x \pm s$ & $t$ & $P$ \\
\hline Negative & 318 & $3.27 \pm 6.65$ & 6.356 & 0.000 \\
\hline Positive & 159 & $10.16 \pm 12.84$ & & \\
\hline
\end{tabular}

\section{Analysis of influencing factors of G17}

The risk factors of $\mathrm{G} 17$ secretion abnormality were analyzed, including sex, age, HP infection, CRP, SAA, ESR. It was found that the risk of abnormal G17 secretion in Hp infected patients was 1.713 times higher than that in uninfected patients, which was 2.692 times in patients with increased SAA. Other factors had no effect on the excretion of G17(Table 4).

Table 4

Analysis of influencing factors of G17

\begin{tabular}{|llll|}
\hline Factors & HR & $95 \% \mathrm{Cl}$ & $\boldsymbol{P}$ \\
\hline Sex & 1.098 & $0.716,1.685$ & 0.699 \\
\hline Ages & 1.018 & $0.995,1.040$ & 0.121 \\
\hline Hp positive & 1.713 & $1.100,2.688$ & 0.017 \\
\hline CRP & 0.552 & $0.285,1.071$ & 0.079 \\
\hline SAA & 2.692 & $1.202,6.028$ & 0.016 \\
\hline ESR & 1.698 & $0.712,4.051$ & 0.233 \\
\hline
\end{tabular}

\section{Discussion}


Gastrin is a gastrointestinal hormone secreted by gastric antrum $\mathrm{G}$ cells, and its level is mainly affected by the number and function of $\mathrm{G}$ cells, as well as by the negative feedback regulation of gastric acid ${ }^{[7]}$. As a gastrointestinal hormone, G17 can not only maintain the integrity of digestive tract structure, but also regulate the function of digestive tract. Gastrin 17 level can be used as a biomarker to reflect the function and structure of gastric mucosa. Combined with serum PG and G17 levels, it can provide diagnostic information of gastric mucosa, reflect the degree of gastric aging too, and distinguish pathological state from healthy state ${ }^{[8]}$. G17 also has multiple biological functions of promoting proliferation and inhibiting apoptosis. The changes of serum G17 level can reflect the state of gastric mucosal lesions, so it can be used as a screening index for gastric cancer and precancerous diseases.

PG is the precursor of pepsin, which is mainly secreted by the main cells. PGI, PGII and their ratio are considered as predictors of various gastric diseases, including atrophic gastritis (AG) and intestinal metaplasia (IM), which are defined as precancerous lesions of gastric cancer ${ }^{[9]}$. One meta-analysis showed that the sensitivity and specificity of PG in the diagnosis of $A G$ were $69 \%$ and $88 \%{ }^{[10]}$, while another meta-analysis for joint detection showed that the sensitivity and specificity were $74.7 \%$ and $95.6 \%{ }^{[11]}$, which were also higher than that of $\mathrm{G} 17$ alone in the diagnosis of $A G^{[12]}$. In this study, G17 and PG showed a weak to moderate correlation, consistent with Wang Rui's result ${ }^{[13]}$, which side confirmed that the combined screening effect of G17 and PG was better.

HP positive is considered as a primary carcinogen. HP infection can induce cell division, increase the probability of cell gene mutation, and play an important role in the occurrence and development of gastric cancer ${ }^{[14]}$. Studies on Helicobacter pylori infection were carried out in China and South Korea respectively. It was found that in Hp positive patients the serum levels of G-17, PGI and PG II increased, especially PG $\otimes$, while the ratio of PGI/PGII decreased ${ }^{[8,15]}$. This study also found that the level of $G 17$ in $\mathrm{Hp}$ positive group was significantly higher than that in negative group, which was showing no difference with other studies ${ }^{[16,17]}$. Patients with Helicobacter pylori infection and low PGI / PGIl ratio should be considered as higher risk of GC and secondary diagnosis (endoscopy and histology) should be performed ${ }^{[18]}$.

Because G17 is easily affected by many factors, such as lesion location, atrophic degree, HP infection, and even inflammatory indicators, for example, this study found that the risk of abnormal G17 in patients with elevated serum amyloid A increased by 2.692 times, so it is not a major diagnostic indicator, but can be combined with PG and Hp detection as a reference to further improve the diagnosis rate of atrophic gastritis and early gastric cancer, also can directly screen high-risk groups. Arrange endoscopy and histological examination according to the results, enhance the compliance of screening and treatment of gastric diseases, avoid unnecessary radiation and discomfort caused by endoscopic examination.

In recent years, Chinese scholars have been drawing on the overseas screening mode of gastric cancer, combined with the situation of China and the current epidemiological investigation, have successively promoted the "four items gastric function" test combined with gastrin-17, pepsinogen, and Helicobacter 
pylori. With expectation to find and optimize the screening path of early gastric cancer suitable for China's national conditions, timely provide evaluation and intervention suggestions for high-risk groups, early screening, early diagnosis, early treatment, reduce mortality, prolong life, and protect people's health.

\section{Conclusions}

The incidence rate and mortality of gastric cancer in China are higher. Precancerous lesions are the key stage of the development of gastric cancer. Therefore, it is necessary to actively find effective means to screen gastrointestinal diseases. Combined screening is helpful to early screen gastrointestinal diseases in healthy people or high-risk groups of gastric cancer, but the influence of relevant factors should be excluded to improve the reliability of the results.

\section{Declarations}

\section{Ethics approval and consent to participate}

The institutional review board of Shanghai Changhai Hospital Ethics Committee approved all procedures.

Consent to Participate All participants were informed about their confidentiality and were asked to voluntarily electronically consent to participate. There was no incentive for participation. "Your involvement in the study is voluntary, and you may choose not to participate or to stop at any time without penalty or loss of benefits to which you are otherwise entitled."

\section{Consent for publication}

Not applicable.

\section{Competing interests}

The authors declare that they have no competing interests.

\section{Funding}

There is no funding to report.

\section{Acknowledgements}

Not applicable.

\section{Availability of data and materials}

All data used to support the findings of this study are included within the article in Figs. All datasets on which the conclusions of the manuscript rely are presented in the paper.

\section{Authors' contributions}


SY, ZJC, XSP and YR designed the experiment; SY, ZJC and YR contributed to drafting and revise the manuscript; SY, ZJC, XSP and YR critically reviewed and revised the manuscript. All authors have read and approved the final manuscript.

\section{References}

1. Bray F, Ferlay J, Soerjomataram I, Siegel RL, Torre LA, Jemal A. Global cancer statistics 2018 : GLOBOCAN estimates of incidence and mortality worldwide for 36 cancers in 185 countries. CA Cancer J Clin. 2018 Nov;68(6):394-424. doi:10.3322/caac.21492.

2. Cheung KS, Chan EW, Wong AYS, Chen L, Wong ICK, Leung WK. Long-term proton pump inhibitors and risk of gastric cancer development after treatment for Helicobacter pylori: a population-based study. Gut. 2018 Jan;67(1):28-35. doi:10.1136/gutjnl-2017-314605.

3. Tu H, Sun L, Dong X, Gong Y, Xu Q, Jing J, et al. Temporal changes in serum biomarkers and risk for progression of gastric precancerous lesions: a longitudinal study. Int J Cancer. $2015 \mathrm{Jan}$ 15;136(2):425-34. doi: 10.1002/ijc.29005.

4. Tu H, Sun L, Dong X, Gong Y, Xu Q, Jing J, et al. A Serological Biopsy Using Five Stomach-Specific Circulating Biomarkers for Gastric Cancer Risk Assessment: A Multi-Phase Study. Am J Gastroenterol. 2017 May;112(5):704-15. doi:10.1038/ajg.2017.55.

5. Corona G, Cannizzaro R, Miolo G, Caggiari L, De Zorzi M, Repetto O, et al. Use of Metabolomics as a Complementary Omic Approach to Implement Risk Criteria for First-Degree Relatives of Gastric Cancer Patients. Int J Mol Sci. 2018 Mar 7;19(3):750. doi: 10.3390/ijms19030750.

6. Roman LD, Lukyanchuk R, Sablin OA, Araslanova El, Eklund C, Hendolin P, et al. Prevalence of H. pylori Infection and Atrophic Gastritis in a Population-based Screening with Serum Biomarker Panel (GastroPanel ${ }^{\circledR}$ ) in St. Petersburg. Anticancer Res. 2016 Aug;36(8):4129-38.

7. Wang Y, Zhu Z, Liu Z, Zhao Z, Xue X, Li X, et al. Diagnostic value of serum pepsinogen I, pepsinogen II, and gastrin-17 levels for population-based screening for early-stage gastric cancer. J Int Med Res. 2020 Mar;48(3):300060520914826. doi:10.1177/0300060520914826.

8. Shan JH, Bai XJ, Han LL, Yuan Y, Sun XF. Changes with aging in gastric biomarkers levels and in biochemical factors associated with Helicobacter pylori infection in asymptomatic Chinese population. World J Gastroenterol. 2017 Aug 28;23(32):5945-5953. doi: 10.3748/wjg.v23.i32.5945.

9. Miki K, Urita Y. Using serum pepsinogens wisely in a clinical practice.J Dig Dis. 2007 Feb;8(1):8-14. doi: 10.1111/j.1443-9573.2007.00278.x.

10. Huang YK, Yu JC, Kang WM, Ma ZQ, Ye X, Tian SB, et al. Significance of Serum Pepsinogens as a Biomarker for Gastric Cancer and Atrophic Gastritis Screening: A Systematic Review and MetaAnalysis. PLoS One. 2015 Nov 10;10(11):e0142080. doi: 10.1371/journal.pone.0142080.

11. Zagari RM, Rabitti S, Greenwood DC, Eusebi LH, Vestito A, Bazzoli F. Systematic review with metaanalysis: diagnostic performance of the combination of pepsinogen, gastrin-17 and anti-Helicobacter 
pylori antibodies serum assays for the diagnosis of atrophic gastritis.Aliment Pharmacol Ther. 2017 Oct;46(7):657-667. doi: 10.1111/apt.14248.

12. Wang X, Ling L, Li S, Qin G, Cui W, Li X, et al. The Diagnostic Value of Gastrin-17 Detection in Atrophic Gastritis: A Meta-Analysis. Medicine (Baltimore). 2016 May;95(18):e3599. doi: 10.1097/MD.0000000000003599.

13. Wang $R$, Chen XZ. Prevalence of atrophic gastritis in southwest China and predictive strength of serum gastrin-17: A cross-sectional study (SIGES). Sci Rep. 2020 Mar 11;10(1):4523. doi: 10.1038/s41598-020-61472-7.

14. Shen Ling, Zhu HC, Yang XL, Ya GW, Zhang YS, Hu MD. Correlation of HP infection and expressions of P73, NF-kb and SOX2 and clinicopathologic features in gastric cancer patients. Chin J Nosocomiol; 28(15): 2261-2264.

15. Kim HY, Kim N, Kang JM, Park YS, Lee DH, Kim YR, et al. Clinical meaning of pepsinogen test and Helicobacter pylori serology in the health check-up population in Korea. Eur J Gastroenterol Hepatol. 2009 Jun;21(6):606 - 12. doi: 0.1097/MEG.0b013e3283086757.

16. Yuan Lin, Ding ZS, Zhang YR, Qi YB, Zhou YL, Li DD, et al. Prevalence of different types of Helicobacter pylori infection in chronic gastric diseases and its influences on gastrin-17 and pepsinogen. J Chin Pract Diagn Ther. 2020;34(4):382-5.

17. Zhu CP, Zhao JY, Qian W, Sun MJ, Liu W, Xu JM, et al. Diagnostic Value of Serum Gastrin-17 for Antral Atrophy under Different Hp Infection Status. Chin J Gastroenterol. 2017;22(5):282-6.

18. Mescoli C, Gallo Lopez A, Taxa Rojas L, Jove Oblitas W, Fassan M, Rugge M. Gastritis staging as a clinical priority. Eur J Gastroenterol Hepatol. 2018 Feb;30(2):125-9. doi:10.1097/MEG.0000000000001015.

\section{Supplementary Files}

This is a list of supplementary files associated with this preprint. Click to download.

- Ethicalapprovalletterback.pdf

- Ethicalapprovalletterfront.pdf 